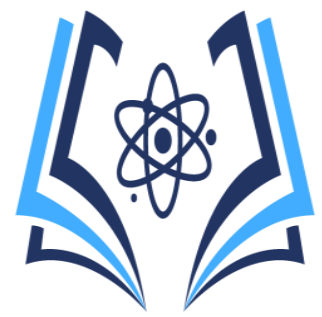

\title{
La automedicación y las consecuencias en la resistencia a antimicrobianos en la población portovejense
}

\section{Self-medication and the antimicrobial resistance consequences in the Portoviejo city habitants}

Prevención de la automedicación

\author{
Alcívar Loor Génessis Daniela, ${ }^{(1)}$ \\ Alfonzo Alcívar Carla Cristina, ${ }^{(2)}$ \\ Lucas Arteaga Karen Monserrate, ${ }^{(3)}$ \\ Jonathan Fonseca Liermo, ${ }^{(4)}$ \\ ${ }^{1}$ Instituto Superior Universitario Portoviejo, Ecuador. Email: danny-ecu @ hotmail.com \\ ${ }^{2}$ Instituto Superior Universitario Portoviejo, Ecuador. Email: ccalfonzo@gmail.com \\ ${ }^{3}$ Instituto Superior Universitario Portoviejo, Ecuador. Email: kar-1995@ hotmail.es \\ ${ }^{4}$ Universidad de La Habana, Facultad de Medicina General Calixto García jonhybarza@gmail.com
}

Contacto: danny-ecu@ hotmail.com

Recibido: 20-07-2021

Aprobado: 02-08-2021

\section{Resumen}

Actualmente, la automedicación es un enigma para el sistema de salud y sus miembros profesionales, debido a que su origen y resolución son complicados, al igual que los beneficios y perjuicios de donde de esta práctica diaria emergen. Se determinan las características y comportamientos que llevan a los usuarios hacia la automedicación para concientización propia de los lectores del presente artículo. La selección de la población fue por muestreo sistemático. Participaron 60 usuarios de la ciudad de Portoviejo, Ecuador. Los resultados determinaron que el $70 \%$ mujeres y $30 \%$ hombres. El $40 \%$. 25 - 31 años adultos jóvenes, $35 \%$ del rango de edad entre 18 - 24 años y por último el límite de 32 años en adelante 25\%. El $70 \%$ asume haber consumido medicamentos estos últimos meses. La frecuencia de consumo de antimicrobianos es $82 \%$ de al menos una vez al mes, $11 \%$ de 2 o 3 veces por semana y todos los días $7 \%$. El $45 \%$ se automedican por los motivos de que cuentan con escaso tiempo para ir al médico, 33\% para dificultad del acceso al servicio de salud $22 \%$ para una existencia de mala calidad para atención al servicio de salud. Para el 92\% no es seguro automedicarse. El 53\% reveló que recomienda medicación a otra persona o familiar y $47 \%$ personas no. Y para el $100 \%$ automedicarse si es dañino para la salud. Llegando a la conclusión que existe un alto porcentaje de recomendación de medicamentos hacia otras personas y de frecuente consumo, los analgésicos.

Palabras claves: Automedicación, consecuencias, Resistencia a antimicrobianos, responsabilidad, conciencia. 
Abstract

Currently, self-medication is an enigma for the health system and its professional members, because its origin and resolution are complicated, as are the benefits and harms from which this daily practice emerges. The characteristics and behaviors that lead users to self-medication are determined for selfawareness of the readers of this article. The selection of the population was by systematic sampling. 60 users from the city of Portoviejo, Ecuador participated. The results determined that $70 \%$ women and 30\% men. 40\%. 25 - 31 years old young adults, $35 \%$ of the age range between 18 - 24 years and finally the limit of 32 years and over 25\%. $70 \%$ assume they have consumed medications in recent months. The frequency of antimicrobial consumption is $82 \%$ of at least once a month, $11 \%$ of 2 or 3 times a week and $7 \%$ every day. $45 \%$ self-medicate for the reasons that they have little time to go to the doctor, $33 \%$ for difficulty in accessing the health service $22 \%$ for a poor quality existence for health service care. For $92 \%$ it is not safe to self-medicate. 53\% revealed that they recommend medication to another person or family member and $47 \%$ people do not. And for $100 \%$ self-medicate if it is harmful to health. Coming to the conclusion that there is a high percentage of recommendation of drugs to other people and of frequent consumption, analgesics.

Keywords: Self-medication, consequences, Antimicrobial resistance, responsibility, awareness.

\section{Introducción}

Actualmente, la automedicación es un enigma para el sistema de salud y sus miembros profesionales, debido a que su origen y resolución son complicados, al igual que los beneficios y perjuicios de donde de esta práctica diaria emergen (1). Es oportuno resaltar que esto puede traer graves problemas para la salud de la persona. Y es que, la automedicación y el abandono de un tratamiento antes de lo indicado por el médico, incrementan el grado de resistencia bacteriana que causan algunas infecciones dentro de las más comunes, la neumonía. En consecuencia, es preciso que mencionar; de varios médicos de la Sociedad Española de Neumología y Cirugía Torácica (SEPAR), quienes alertan del uso inadecuado que se hace en España de los antibióticos aumenta los riesgos de que el tratamiento se vea afectado y fracase.

En un estudio se revela que más del $50 \%$ de los medicamentos se recetan, expenden y venden de manera inadecuada y la mitad de los usuarios los ingieren de una forma incorrecta. Su uso excesivo, insuficiente o indebido tienen efectos adversos para los usuarios y da lugar un gasto innecesario de recursos. Habitualmente todo occidente tiene acceso a una medicina que le alivie el dolor de cabeza, disminuya el estrés, su estado depresivo o la ansiedad que no falta en estos días por las rutinas de la vida actual. La automedicación es una práctica muy peligrosa en cuyas consecuencias puede haber; enmascaramiento de una enfermedad, aparición de reacciones adversas e interacciones medicamentosas, aumento de la resistencia a antimicrobianos, elección incorrecta de medicamentos y riesgo de abuso o hasta farmacodependencia (2).

A nivel mundial, múltiples organizaciones pretenden posicionar a la automedicación como el primer paso en la atención primaria, sin embargo, la línea entre la seguridad y la presencia de eventos adversos es muy delgada. Según criterios valorativos, se destaca que: "el problema sucede cuando las personas se automedican porque han escuchado que ese fármaco le ha funcionado a un conocido. Así los individuos toman decisiones que no son las adecuadas. Debemos acercarnos a la población para decirle cómo administrarse. Cuando una persona asiste a un centro de salud el personal profesional sí que aporta una serie de consejos "tienes que tomar esto" o "no tienes que hacer caso de los que te diga equis persona". Los malos hábitos surgen cuando oyen que le sientan bien 
Periodo. Julio - Diciembre 2021

Vol. 5, Nro. 2, Publicado: 2021-12-31

el medicamento a una amiga o amigo, lo compran en la farmacia y lo toman. Se cree que la automedicación es un fenómeno complicado, además desde el ámbito sanitario manifiesta que antes de tomar medicación se recomienda el uso de medidas no farmacológicas. El problema es que la población, en muchas ocasiones, no lo acepta" (3).

En un sentido amplio, la automedicación implica el consumo de cualquier tipo de medicamento, sin importar su origen, ya sean sintéticos, herbolario u homeopático, pero el acto de la automedicación abarca todo aquello que altere la prescripción realizada por un médico, es decir, la adquisición, uso de medicamentos de casa u otro lugar, interrupción o prolongación de un tratamiento o el incremento y disminución de las dosis. Desde esta perspectiva de análisis la automedicación es un problema social practicado por un alto porcentaje de la población cada día, en todo el mundo y a veces incluso con la ayuda de un profesional de la salud (1).

Desde el punto de vista nacional, el Ecuador no se encuentra exento de la presente problemática, pues no se tiene fácil acceso a los sistemas de salud pública, pese a la gratuidad que ofrece el Ministerio de Salud Pública (MSP); conjuntamente por el alto costo que significa asistir al sector privado. Circunstancia que ha inducido que las personas busquen alternativas que los ayude a mejorar su estado de salud lo más rápido posible, optando por automedicarse; evitan acudir al médico porque para la población implica pérdida de tiempo y dinero. Es por esto que los profesionales de la salud deben enfrentarse dos grandes y desafiantes aristas: La primer arista es concientizar a los usuarios sobre la automedicación, fomentando estilos de vida saludable y promocionando la consulta médica pertinente; y la segunda, demostrar todas las posibles consecuencias que conlleva una negligencia médica, por contar con un inadecuado manejo de tratamientos por parte del personal médico, quien es el que puede prescribir y dosificar los fármacos según la gravedad de los síntomas, la edad, el peso y el horario. Si no se cumple con estos elementos, surgen en la población problemas de salud como: sobredosis, intoxicaciones, enmascaramiento de enfermedades y en el peor de los casos la muerte.

Cuando las personas se preguntan ¿qué medicina tomar?, los analgésicos, antibióticos o antiinflamatorios son las respuestas más comunes. En el área de emergencias en el Hospital Metropolitano de Quito, casi el 90\% de los pacientes que llegan a la atención de emergencia ya se han automedicado. Es recomendable tomar un antibiótico para controlar el dolor, pero solo en el lapso de 24 horas, hasta ser evaluado por un médico. Aplicar calor o frío en la zona del dolor es otra forma de calmarlo, los botiquines deben ser usados en caso de emergencia. Deben contener productos de primeros auxilios que controlen el dolor hasta que llega la atención médica. Pero no se debe usarlos como primera opción de alivio de enfermedades, ni abusar de los medicamentos (4).

Así, se manifiestan cuestiones con respuestas parciales: ¿cuál es el origen de la automedicación?, ¿cuán frecuente es su práctica?, ¿quién lo realiza asiduamente?, ¿los beneficios superan los efectos nocivos que pueda producir?, ¿existen estrategias que sean efectivas para evitarla? Y de esta manera emergen preguntas ante la complejidad de este laberinto de gran debate social.

El objetivo de la investigación es: determinar las características y comportamientos que llevan a los usuarios hacia la automedicación para concientización propia de los lectores del presente artículo.

\section{Materiales y métodos}

Se realizó un estudio no experimental de tipo descriptivo, prospectivo, transversal, de campo y bibliográfico puesto que permite recopilar datos, información y documentos teóricos como son los 
Periodo. Julio - Diciembre 2021

links, artículos científicos, revistas actualizadas, Vol. 5, Nro. 2, Publicado: 2021-12-31 libros en relación con el tema de estudio como aporte a la fundamentación del trabajo de investigación.

El tiempo de ejecución del estudio fue en el período comprendido desde mayo hasta octubre del año 2020 en los habitantes de la ciudad de Portoviejo, provincia de Manabí, República del Ecuador.

En la investigación se tomaron como muestra 60 personas de sexo masculino y femenino mayores de 18 años con el propósito de determinar el nivel de conciencia y conocimiento de los problemas de automedicación y sus consecuencias en la resistencia a antimicrobianos, ya que es un hábito común en la sociedad y no está exento de riesgos: se consumen medicamentos para: el dolor de cabeza, problemas gástricos, estrés, la tos, la alergia; y el fin es de prevenirlos fomentando el recurrir al consultorio médico, al padecer alguna dolencia. Porque el solo hecho que el paciente ingiera la medicación sin indicaciones puede ocasionar efectos nocivos pueden llegar a vulnerar la salud.

Las variables incluidas en el estudio fueron: sociodemográficas (sexo y edad), conocimiento y actitudes frente a la automedicación con analgésicos, antiinflamatorios y antibióticos.

Para la recolección de datos se utilizó la técnica de la encuesta online por medio de Google Forms, con 13 preguntas garantizando la calidad de la información la cual fue anónima y ningún dato permitía identificar a la persona que la había diligenciado; el uso de la información fue solo con fines de conocimiento social, informativo y educativo.

Las fuentes de información que permitieron el respaldo de la presente investigación son de absoluta seguridad la cual se distinguen de la siguiente manera como: Fuentes primarias como revistas científicas (scielo.org redalyc.org, Google Académico, Elsevier), artículos científicos, MSP del Ecuador, Organización mundial de la salud (OMS) y Organización Panamericana de la salud (OPS) y;

fuentes secundarias como investigaciones ya realizadas por otras instituciones.

Entre los métodos que se empleó estuvo el inductivo - deductivo desde la teoría, para la concreción de información relacionada con cada una de las variables de la investigación, mediante la observación y análisis de los hechos llegar a los resultados y sus respectivas conclusiones; y métodos estadísticos, debido a que los resultados de las encuestas estudiadas se tabularon de acuerdo a los datos empíricos.

El tipo de investigación es de carácter cuali cuantitativa, pues se realizó una observación de la población en estudio con sus principales características y del análisis de los datos estadísticos obtenidos.

En el aspecto ético, la investigación se realizó bajo los principios de ética profesional, aplicados por normas de investigación a la población, clasificado como de bajo riesgo, previa explicación y del consentimiento para salvaguardar la confidencialidad de los participantes y la responsabilidad de los investigadores; el estudio no presentó ningún tipo de conflicto de interés.

Entre los recursos para poder llevar a cabo el estudio se contó con los humanos tales como los miembros autores de la investigación y habitantes de la ciudad de Portoviejo; y con los tecnológicos mediante el uso de internet, calculadora, laptop o computadora con programas de Windows SmartScreen, Microsoft Word 2013 para procesar el texto y Microsoft Excel 2013 para tabulación de datos y gráficos de los resultados, además de las redes sociales como Facebook y WhatsApp para difundir el link de la encuesta. Los gráficos que se utilizaron fueron de barras para las muestras de datos cuali-cuantitativos de los habitantes seleccionados, con ello su respectiva discusión.

\section{Resultados}


La encuesta aplicada a la muestra contó con la colaboración de 42 mujeres (70\%) y 18 hombres (30\%) para un total de 60 personas, donde ha sido de mayor relevancia el sexo femenino, en cuanto a las opiniones.

Se evidenciaron dentro de las características socio epidemiológicas con respecto a las edades entre las más frecuentes fue el rango de 25 - 31 años con 24 personas (40\%), definiéndose usuarios adultos jóvenes propensos a la automedicación. En segundo lugar, se encuentran 21 personas $(35 \%)$ del rango de edad entre 18 - 24 años mostrándose casi representativa con el rango de edad más relevante, y por último el límite de 32 años en adelante, considerándose una edad menos propensa a automedicación, dado el nivel de experticio ante el consumo de medicamentos de manera no recomendada por los facultativos.

Consumir algún tipo de medicamento estos últimos meses ha sido de mayor significancia en 42 personas (70\%) y 18 personas (30\%) alegaron que no.

En cuanto al tipo de medicamentos más frecuentes, que consumen los usuarios cuando padecen alguna dolencia son los analgésicos (diclofenaco, paracetamol), referido por 45 personas (75\%). En este orden de análisis, los medicamentos se expenden en las farmacias como venta libre por eso el alto porcentaje, siguiéndole los antiinflamatorios como el ibuprofeno donde 12 personas (20\%) alegaron sobre este particular, este medicamento a lo largo de la vida puede causar problemas renales importantes.

En consecuencia, los antibióticos (Amoxicilina, ciprofloxacino, cefalexina, azitromicina) son consumidos indiscriminadamente por 3 personas (5\%) demostrando que esta parte de la muestra puede ser propensa a resistencia a los antimicrobianos.

La frecuencia de consumo con que la mayoría de la muestra hace el uso de antimicrobianos, es decir, 49 personas $(82 \%)$ es de al menos una vez al mes, el segundo puesto están 7 personas $(11 \%)$ de 2 o 3 veces
Vol. 5, Nro. 2, Publicado: 2021-12-31

por semana y todos los días con el total de 4 personas (7\%).

En esta lógica, salió como relevante que 27 personas $(45 \%)$ encuestadas del total de la muestra respondieron que se automedican por los motivos de que cuentan con escaso tiempo para ir al médico, el segundo más relevante son 20 personas (33\%) que presentan dificultad para el acceso al servicio de salud por lo que se demoran 2 días para acatar un turno médico y el papeleo. En cambio 13 personas $(22 \%)$ aluden a que existe una mala calidad para atención al servicio de salud.

La automedicación no es una práctica segura según refiere las 55 personas (92\%) del estudio y $5(8 \%)$ personas mencionan que sí lo es.

Según la muestra estudiada 48 personas (80\%) mencionan que los antimicrobianos si producen resistencia, es decir, están conscientes de los daños que producen cuando se abusan de estos medicamentos cuando se compran sin prescripción médica en especial la Amoxicilina. En cuanto a los $12(20 \%)$ restantes exclamó que no producen resistencia.

En los 36 de los encuestados (60\%) revelan que cuando sienten que presentan síntomas de malestar, Si acude al médico; en cambio 24 personas (40\%) menciona que No acude. Se puede notar una relevancia en las respuestas positivas.

El $36 \%$ la muestra consideró que, Si continuará automedicándose, mientras que el $64 \%$ de los estudiados manifestó que No lo hará, lo cual tuvo mayor destacamento entre las respuestas dirigidas.

Se vislumbraron que en la muestra tomada de 60 personas $(100 \%), 47 \quad(78 \%)$ individuos si acostumbran leer los insertos que hay dentro de las cajas de los productos médicos, mientras tanto que 13 personas $(22 \%)$ No lo leen demostrando que no prestan interés de los compuestos o reacciones que producen los medicamentos cuando los toman. 
Periodo. Julio - Diciembre 2021

Vol. 5, Nro. 2, Publicado: 2021-12-31

En los que corresponde a las recomendaciones de fármacos de los encuestados hacia otras personas, 32 $(53 \%)$ revelaron que ellos les brindan los medicamentos que ya no ocupan a las personas que lo necesitan sin consentimiento médico. Se usa de manera empírica el sistema placebo convirtiéndolo en un motivo más para la automedicación. En tanto que, $28(47 \%)$ personas no recomiendan sus medicamentos a nadie sin que un médico lo oriente. Y finalmente, Automedicarse Si es dañino para la salud menciona la muestra total de 60 personas (100\%) seleccionada del estudio, lo cual significa que están conscientes de que se siguen automedicando sin prestar atención a las consecuencias que puede llevar a lo largo de la vida con este hábito poco saludable.

\section{Discusión}

En la provincia de Manabí existen y perduran problemáticas en el área de la salud de la población; tanto en las regiones urbanas, como rurales y periféricas. Los principales problemas a atender son: expendio de medicamentos sin receta médica, automedicación, escaso conocimiento de los principios activos de los medicamentos, desconocimiento de la acción del medicamento sobre un padecimiento agudo o crónico; así como en patologías determinadas, aversión a las consultas médicas, carente cultura ante la tenencia de un botiquín en caso de emergencias, combinación de medicamentos naturales con medicamentos de origen químico o biológico.

Portoviejo, se encuentra ante los mismos problemas que se demuestran en la provincia, adicional a la carente cultura de los adultos de mantener los medicamentos fuera del alcance de los niños. Se ha manifestado una tendencia desde 2010 a la automedicación y hoy día se mantiene. De igual modo se han revelado casos de intoxicaciones por productos agrícolas y por picaduras de insectos; a lo que los usuarios han respondido con cualquier medicamento provocando reacciones no tan beneficiosas. En relación con los antibióticos ocurre algo similar, creándose resistencia por la interrupción de los ciclos dentro del tratamiento o por consumirlos sin realizarse antibiogramas; sobre todo en casos de mayor necesidad.

En el estudio de pertinencia de farmacia por parte del Instituto Superior Tecnológico "Portoviejo" menciona; una de las dificultades más graves que se evidencian en el ámbito profesional son: la venta de medicamentos sin prescripción médica, la sustitución de medicamentos por otros sobre la base de la razón de la persona que expende los medicamentos, venta a niños, orientaciones erróneas con relación a los criterios de un médico (5).

Según el estudio efectuado por investigadores de la Universidad Rey Juan Carlos de Madrid, el 20,17\% de los españoles toma algún medicamento sin prescripción del facultativo, siendo las mujeres las que más practican la automedicación, concretamente el $16,93 \%$ de las mujeres por el $14,46 \%$ de los hombres (6). Puede afirmarse que la investigación es congruente con el actual estudio realizado por las autoras donde contó con el $70 \%$ mujeres y 30\% de hombres y consecuentemente ha sido de mayor relevancia el sexo femenino, en cuanto a las opiniones.

La automedicación es la ingestión de medicamentos por decisión propia y sin receta o control médico, que generalmente en países desarrollados es el $60 \%$ de la población la que practica la misma. Las medicinas que tratan condiciones crónicas o agudas, tales como presión arterial elevada o diabetes, el consumo sin prescripción médica es menor, mientras que lo contrario ocurre para los medicamentos que tratan condiciones menores, como tratamientos de síntomas como cefalea, gripe, tos, fiebre.

Según una investigación realizada en Paraguay sobre las características clínicas de la automedicación en adultos de tres Unidades Médicas de Salud Familiar el $66 \%$ de los pacientes consumía analgésicos para 
Periodo. Julio - Diciembre 2021

Vol. 5, Nro. 2, Publicado: 2021-12-31

calmar cualquier dolencia (7). Consecuentemente, en el estudio de automedicación de analgésicos y antibióticos en estudiantes de pregrado de medicina revela que el $84 \%$ de las mujeres y $81 \%$ de hombres consumen analgésicos con mayor frecuencia que de otro medicamento (8).

Se coincide con los autores de dichas investigaciones donde el tipo de medicamentos más frecuentes que consumen los usuarios del presente estudio cuando padecen alguna molestia general; son los analgésicos como diclofenaco y paracetamol, referido por el $75 \%$ de las personas, luego a los antiinflamatorios como el ibuprofeno mostrando el $20 \%$ de las opiniones, este último medicamento si se abusa de su consumo puede llevar a la persona a sufrir de graves problemas gástricos y renales.

Por otro lado, los antibióticos (Amoxicilina, ciprofloxacino, cefalexina, azitromicina) son consumidos abusivamente por el (5\%) de los usuarios demostrando que esta parte de la muestra puede ser propensa a resistencia a los antimicrobianos y el paciente necesitará cada vez de dosis o medicamentos de mayor espectro para combatirla. Cabe destacar que el consumo de antimicrobianos sin receta médica tiene una profunda relación con el hecho de haber sido curado antes por los mismos (9). La edad para la ingestión de medicamentos sin control médico es variable, pero, existe una constante según explica un estudio acerca del Perfil de automedicación en funcionarios de una industria farmacéutica donde las edades que frecuentemente se automedican están entre los 20 a 30 años con una cifra del 60\% (2), es decir una población adulta joven, estos datos son significativos para el presente estudio, los resultados de la encuesta arrojaron también valores relevantes entre las edades de 25 - 31 años con el $40 \%$ de las personas.

Un punto de vista de referencia en el estudio de la Universidad San Sebastián sobre la encuesta del consumo de medicamentos; en los últimos 30 días el
$72 \%$ de la población alegó que si lo había hecho mientras tanto que el $28 \%$ optó por el no (10). El análisis anterior permite reconocer que los resultados de la investigación presenten son de correspondencia dado que consumir algún tipo de medicamento estos últimos meses ha sido de mayor significancia en el $70 \%$.

Estudios previos como la automedicación en estudiantes de una residencia universitaria en Chillán de Chile han hallado que el $80 \%$ considera que automedicarse es una práctica riesgosa y a su vez el $64 \%$ ha recomendado algún medicamento (11). En congruencia con las ideas antes expresadas se desvela una coincidencia en dos de las 13 preguntas aplicadas en la encuesta; donde se demuestra que la automedicación no es una práctica segura según refiere el $92 \%$ del estudio; en tanto a las recomendaciones de fármacos de los encuestados hacia otras personas, $53 \%$ revelaron que ellos si sugirieron medicamentos que a ellos les hizo buen efecto y alivió su dolencia añadida a que también brindaban los que ya no ocuparon.

El incremento de la resistencia a los antimicrobianos es un problema inevitable que de no combatirse a tiempo puede complicar la salud de las futuras generaciones, con un retorno a la era preantibiótica (12). La investigación de (8) afirma que el $93 \%$ de las personas encuestadas mencionan que automedicarse con antibióticos produce resistencia antimicrobiana, se encontró similitud con la autora de la investigación donde la muestra estudiada el $80 \%$ señalan que los antimicrobianos si producen resistencia, es decir, existe de los daños nocivos que se provoca cuando se realiza el uso indiscriminado de estos medicamentos cuando se compran sin prescripción médica en especial la Amoxicilina, que debería de ser de expendio con receta médica.

Cuán importante es leer siempre el inserto del medicamento para saber cuáles son las reacciones adversas conocidas y acudir al médico (13). Es 
oportuno resaltar que el 78\% si acostumbran leer los insertos de los productos médicos, mientras que $22 \%$ no. Estos datos son congruentes con la autora Berrouet lo que comprueba que las personas son conscientes de su automedicación y de los efectos adversos que pueden producirse en su organismo.

En este estudio, las principales razones de automedicación concuerdan con una investigación realizada en Cartagena, Colombia sobre automedicación y creencias en torno a su práctica donde arrojó que el 28,3\% de os usuarios lo hacía por la falta de tiempo para consultar en una institución de salud, la tardanza o demora en la atención por el $22,7 \%$, y por la dificultad en el acceso a las instituciones de salud con relación al lugar de residencia dado por el 21,3\% (14). Demostrando así la similitud de cifras relevantes donde el $45 \%$ de las personas encuestadas respondieron que se automedican por los motivos de que cuentan con escaso tiempo para ir al médico, el segundo más relevante fue el $33 \%$ que presentan dificultad para el acceso al servicio de salud por lo que se demoran dos días para tomar un turno médico y el trámite.

En el estudio de las actitudes, conocimientos y prácticas frente a la automedicación con productos herbales y psicofármacos en estudiantes de medicina de Medellín-Colombia el $78.6 \%$ afirmó que continuaría automedicándose (15), sin embargo, en esta investigación los datos de la actual encuesta se contraponen a la idea antes mencionada indicando $64 \%$ de los estudiados manifestó que no lo seguirá haciendo, y en relación con esta posición la actitud no es segura puesto que puede volver a hacerlo.

Finalmente, el $60 \%$ de la muestra señala que cuando sienten síntomas de malestar, si acude al médico, esta afirmación se contrapone a las ideas antes expuestas sobre automedicación. Así mismo donde el 100\% de los encuestados menciona que automedicarse si es dañino para la salud y representa graves consecuencias para el organismo humano.

\section{Conclusiones}

Vol. 5, Nro. 2, Publicado: 2021-12-31

Con los resultados dados en este estudio se dio respuesta al objetivo planteado por lo que se describieron características sociodemográficas (sexo y edad), conocimiento y actitudes frente a la automedicación con analgésicos, antiinflamatorios y antibióticos con sus consecuencias entre los habitantes de la ciudad de Portoviejo, Ecuador.

Existe un alto porcentaje de recomendación de medicamentos hacia otras personas y de frecuente consumo de analgésicos sin prescripción médica.

La muestra de estudio señaló que no seguirá automedicándose, pero no es una actitud segura para volverlo cometer.

Las personas recurren a la automedicación cuando consideran que el problema de salud no es grave, por falta de dinero, por falta de tiempo o por falta de credibilidad en el sistema de salud o el médico, por lo tanto, los esfuerzos se deben dirigir a la educación de los pacientes y farmacéuticos sobre todo en el uso de medicamentos de venta libre.

La utilización de antibióticos a través de la automedicación para enfermedad de origen viral o de bacterias, trae como consecuencia el que los antibióticos puedan dejar de ejercer su efecto cuando sean necesarios, por tal motivo es recomendable que se consulte al médico él recomendara cual será el mejor tratamiento dependiendo del grado y tipo de enfermedad que presente y de ese modo evitar cualquier tipo de reacción perjudicial para la salud y vida.

La automedicación es inevitable, sin embargo, es necesario crear el nivel de conciencia necesario para que las personas puedan percatarse que una inadecuada administración de medicamentos a través de la automedicación pueda ocasionar graves daños a la salud.

Se debe realizar una investigación más afondo sobre la automedicación con plantas herbales y sus efectos con otros medicamentos. 
Los medicamentos curan y alivian patologías que alteran la homeostasis del organismo humano, pero suponen de riesgos y costes en los que no se debe pasar por alto. Son herramientas que van en pro de la salud humana pero no la única.

Los problemas de la salud de hoy en día sostienen un concepto de prevención social, en opinión de las autoras esta estrategia debe fomentarse al mismo tiempo que se realiza una investigación.

Referencis Biliográficas

Chávez AH, Sesma ARM. Farmacología general. Una guía de estudio. [Online].; 2017. Available from: https://accessmedicina.mhmedical.com/content.aspx ?bookid=1489\&sectionid=96952696.

Raquel Cuevas LSPAMGDGLGMG. Perfil de automedicación en funcionarios de una industria. [Online].; 2019. Available from: http://scielo.iics.una.py/pdf/iics/v17n1/1812-9528-iics-17-01-99.pdf.

Luis Mañas Viniegra SMCEMR. Automedicación: Inconvenientes, consecuencias y posibles soluciones. [Online].; 2018. Available from: https://books.google.com.ec/books?id=0C-

ZDwAAQBAJ\&pg=PT148\&lpg=PT148\&dq=Elisa+Ferrer+explica+que:+\%E2\%80\%9Cel+problema+sucede+c uando+las+personas+se+automedican+porque+han+escuchado+que+ese+f\%C3\%A1rmaco+le+ha+funcionado+ a+un+conocido.+As\%C3\% AD+los+individ.

Castro M. ¿Qué medicina tomar? La mala idea de automedicarse. [Online].; 2019. Available from: https://gk.city/2019/08/10/que-medicina-tomar-automedicacion/.

Instituto Tecnológico Superior Portoviejo. Estudio de pertinencia en las farmacias. SInapsis. 2019;: p. 1-8.

Comisión Europea. Las mujeres se automedican más que los hombres, según una investigación española. [Online].; 2020. Available from: https://cordis.europa.eu/article/id/33315-spanish-research-shows-women-aremore-likely-to-selfmedicate-than-men/es.

Nicolás Emilio Real Aparicio DSB,FC,ESLA. Características clínicas de la automedicación en adultos de tres Unidades de Salud Familiar de Paraguay en 2019. [Online].; 2020. Available from: http://scielo.iics.una.py/pdf/spmi/v7n1/2312-3893-spmi-7-01-77.pdf.

Marie Claire Berrouet Mejía MLRDRB. Automedicación de analgésicos y antibióticos en estudiantes de pregrado de medicina. [Online].; 2017. Available from: https://www.redalyc.org/jatsRepo/1590/159052395003/html/index.html4.

Blanca Obón-Azuara ÁGGIGCCTA. Percepción asociada a la automedicación con antimicrobianos en farmacias de Fortaleza, Brasil. [Online].; 2019. Available from:

https://www.ncbi.nlm.nih.gov/pmc/articles/PMC7025993/pdf/main.pdf.

Morales AM. Universidad de San Sebastián. [Online].; 2017. Available from:

http://www.ipsuss.cl/ipsuss/site/artic/20171129/asocfile/20171129133304/encuesta_salud_11_2017_prensa_.pdf. 
Valeria Altamirano Orellana KHGEMCFMMIMOEMSARF. Automedicación en estudiantes de una residencia universitaria en Chillán, Chile. [Online].; 2019. Available from:

https://www.scielosp.org/article/rcsp/2019.v45n1/e1189/\#.

Teresa Camou PZMH. Revista Médica de Uruguay. [Online].; 2017. Available from:

http://www.scielo.edu.uy/pdf/rmu/v33n4/1688-0390-rmu-33-04-00104.pdf.

Grupo Mediforum. Salud. [Online].; 2020. Available from: https://www.consalud.es/pacientes/efectos-adversosinsospechados-la-importancia-prospecto-medicamento_48117_102.html.

Moraima DTR, Anderson DP, Zorayda BP, Irma CÁ. Revista Cuidartte. [Online].; 2017. Available from: https://www.redalyc.org/articulo.oa?id=359549159010.

Marie Claire Berrouet Mejía MLRDRB. Actitudes, conocimientos y prácticas frente a la automedicación con plantas herbales y psicofármacos. [Online].; 2018. Available from: https://core.ac.uk/download/pdf/297173881.pdf. 